\title{
Universiteit
}

Leiden

The Netherlands

\section{Psychometric properties of the adolescents' interpretation and belief questionnaire (AIBQ) for measuring interpretation bias in Chinese adolescents}

Yu, M.; Westenberg, P.M.; Wang, Y.; Wang, J.P.; Miers, A.C.

\section{Citation}

Yu, M., Westenberg, P. M., Wang, Y., Wang, J. P., \& Miers, A. C. (2021). Psychometric properties of the adolescents' interpretation and belief questionnaire (AIBQ) for measuring interpretation bias in Chinese adolescents. Current Psychology.

doi:10.1007/s12144-021-01346-x

Version: $\quad$ Publisher's Version

License: $\quad$ Licensed under Article 25fa Copyright Act/Law (Amendment Taverne)

Downloaded from: https://hdl.handle.net/1887/3249366

Note: To cite this publication please use the final published version (if applicable). 


\title{
Psychometric properties of the adolescents' interpretation and belief questionnaire (AIBQ) for measuring interpretation Bias in Chinese adolescents
}

\author{
Meng $\mathrm{Yu}^{1,2} \cdot$ P. Michiel Westenberg ${ }^{2} \cdot \mathrm{Ye} \mathrm{Wang}^{3} \cdot$ Jianping Wang ${ }^{1}$ (D) $\cdot$ Anne C. Miers $^{2}$ \\ Accepted: 7 January 2021 \\ (C) The Author(s), under exclusive licence to Springer Science+Business Media, LLC part of Springer Nature 2021
}

\begin{abstract}
The cognitive model of social anxiety disorder hypothesized that socially anxious individuals tend to negatively interpret ambiguous scenarios, which was termed an Interpretation bias (IB). The Adolescents' Interpretation Bias Questionnaire (AIBQ) is a widely used self-report instrument to measure IB towards social situations for adolescents. The main objective of the current study was to culturally revise AIBQ in Chinese adolescents. In total, 960 adolescents participated, and four weeks later, 185 of them were re-measured with Chinese version of AIBQ (C-AIBQ). We tested construct validity, examined internal consistency and test-retest reliability, and assessed convergent and divergent validity of the C-AIBQ subscales. Overall, C-AIBQ variables showed satisfactory construct validity, internal consistency, test-retest reliability and convergent and discriminant validity, in particular the social negative interpretation subscale. Our results indicate that this instrument is adequate to measure interpretation bias in Chinese adolescents.
\end{abstract}

Keywords Chinese adolescents $\cdot$ Cultural validation $\cdot$ Interpretation bias $\cdot$ Psychometric properties $\cdot$ Social anxiety

\section{Introduction}

The essential characteristic of social anxiety disorder (SAD) is a marked fear or significant anxiety in social interactions in which the individual is potentially observed or scrutinized by others (DSM-5; American Psychiatric Association, 2013). It is one of the most prevalent mental health disorders in youth (e.g., Kessler et al., 1994), with prevalence rates of about $13 \%$ in studies with Western samples (e.g., Kessler et al., 1994). The presence of SAD during adolescence is associated with impairments in academic (e.g., Blöte, Miers, Heyne, \& Westenberg, 2015) and social functioning (e.g., Blöte \& Westenberg, 2007; La Greca \& Lopez, 1998). The national

Jianping Wang

wjphh@bnu.edu.cn

1 Beijing Key Laboratory of Applied Experimental Psychology, Faculty of Psychology, Beijing Normal University, Beijing, People's Republic of China

2 Institute of Psychology, Developmental and Educational Psychology Unit, Leiden University, Leiden, Netherlands

3 Changzhou ART Vocational College of Jiangsu Province, Changzhou, People's Republic of China prevalence rate of SAD in Chinese youth is not yet available, however, a few transcultural studies have indicated that, compared to Western samples, Chinese adolescents tend to report a higher level of social fear (Miers, Blöte, Bögels, \& Westenberg, 2008; Yu, Westenberg, Li, Wang, \& Miers, 2019; Zhou, Xu, Inglés, Hidalgo, \& La Greca, 2008). This calls for a psychometrically sound instrument to measure interpretation bias, a key mechanism in the maintenance of social anxiety, in Chinese adolescents.

According to the cognitive model for social anxiety disorder, developed by Clark and Wells (1995), socially anxious individuals tend to interpret ambiguous scenarios in a negative or threatening manner, which is termed Interpretation bias (IB). These negative interpretations refer to the perspective of others (Hirsch, Meeten, Krahé, \& Reeder, 2016), for instance, with regard to a no-question-asking situation after a speech, a socially anxious adolescent might ponder "They did not think my presentation was interesting" (Miers et al., 2008). In addition to the field of social anxiety, interpretation bias is also found to be a significant feature in adolescents with depressive emotion (or a diagnosis of major depression) (Sfärlea et al., 2020; Songco, Booth, Spiegler, Parsons, \& Fox, 2020), and a traumatic experience (Mueller et al., 2020). In these populations the interpretation bias refers to stimuli related to the specific problem, for example, adolescents who have 
experienced trauma interpret ambiguous words as related to the trauma rather than as neutral. Whilst the tendency to negatively interpret stimuli has been found in several psychopathological syndromes, the majority of research concentrates on interpretation bias related to social anxiety.

During social occasions, including anticipating to enter or actually being in one, the incompleteness and the ambiguity of social cues highlights the inevitability that people explain the situational cues in their own way. The way people interpret ambiguous situations can directly impact on their subsequent reaction or behavior, especially for those with a high level of social anxiety. Furthermore, during adolescence, individuals' attention shifts to peer intimacy and identity (Meacham \& Santilli, 1982). This might mean that, particularly in this life-stage, an interpretations bias could interfere negatively with peer relationships (Miers, Sumter, Clark, \& Leigh, 2020). Hence, it is necessary to research interpretation bias for social situations in adolescents.

There is evidence that the cognitive model for SAD, originally developed to explain social phobia in adults, also applies to adolescents (Hodson, McManus, Clark, \& Doll, 2008). Empirically, both Western and Chinese literature has demonstrated that socially anxious adolescents are inclined to make more negative interpretations of social information and stimuli (e.g., China, Yu et al., 2019; Italy, Giannini \& Loscalzo, 2016; Netherlands, Miers et al., 2008; U.K., Creswell, Murray, \& Cooper, 2014), indicating the presence of such an interpretation bias towards social situations. Previous studies from Western samples have consistently shown the association between interpretation bias and social anxiety. Nevertheless, among published research, only Yu et al. (2019) extended IB and social anxiety research to the Chinese culture, and found evidence for high socially anxious adolescents as being more likely to interpret ambiguous social situations negatively compared to non-socially anxious adolescents. However, so far, a sound instrument to measure interpretation bias has not yet been properly validated for Chinese adolescents. Those that are available lack convergent and discriminant validity (Zhu, 2016), or have only revised the instruments for youth populations without enough information about reliability and validity (Fu, Du, Au, \& Lau, 2013; Yang, 2011).

In the present study, we chose the Adolescents' Interpretation Bias Questionnaire (AIBQ). Although a few tools have been developed and adopted to measure youth's interpretation bias (e.g., Reuland \& Teachman, 2014), the AIBQ is the only self-report instrument available in the literature to measure interpretation bias towards social situations in adolescents. In addition, it includes different IB components: negative and positive interpretations, and negative belief, in both social and non-social situations. Miers et al. (2008) developed the AIBQ that includes five ambiguous social situations and five non-social situations, and assesses negative, positive, and neutral interpretations. Adolescents are asked to rate how likely each interpretation would appear in their mind and to report which interpretation would be the most believable for them regarding each ambiguous scenario.

Research using the AIBQ to measure interpretation bias has shown that compared to non-anxious groups, socially anxious adolescents report more negative interpretations and negative belief of ambiguous social situations (Giannini \& Loscalzo, 2016; Miers et al., 2008; Yu et al., 2019). In addition, a negative interpretation bias also differentiated between adolescents following a high social anxiety developmental trajectory from adolescents following a moderate social anxiety trajectory (Miers, Blöte, Rooij, Bokhorst, \& Westenberg, 2013). Furthermore, researchers suggest that negative belief of social situations could be a clinical feature of social anxiety (Loscalzo, Giannini, \& Miers, 2017), and the social negative interpretation subscale showed its optimum cut-off for social anxiety disorder at a score of 3.5 (possible range 1-5) in Italian adolescents (Loscalzo \& Giannini, 2015).

The objective of the current study was to culturally revise the AIBQ for Chinese adolescents (C-AIBQ), and to investigate its psychometric properties. We examined i) the construct validity of negative interpretations and positive interpretations, both in social and non-social situations; ii) the reliability of negative interpretations and positive interpretations both in social and non-social situations; and reliability of negative belief subscales in social and non-social situations; and iii) convergent and discriminant validity of C-AIBQ subscales through correlations with social anxiety, and depression, respectively.

\section{Methods}

\section{Participants}

In total, 983 participants were recruited from one middle school in Nanjing, China; 23 of these were excluded from analyses (see the exclusion procedure in the data analysis section), leaving 960 as the final T1 sample (504 boys and 450 girls, with six participants did not report their gender information), with an age range of $11-19$ years $(M \pm S D=15.41 \pm$ $1.51)$. The majority $(60.5 \%)$ of adolescents had a senior secondary education background (equivalent to 10th to 12th American grades), $39.5 \%$ of students reported a junior secondary education (7th to 9th grade). Most of the participants (65.7\%) reported that their parents were married, $7.0 \%$ was divorced, $1.6 \%$ was separated, and $0.8 \%$ was widowed, and $24.9 \%$ did not report the parental marriage status. According to the rule of the administrative division of China, $63.6 \%$ reported their place of residence was a city, $5.0 \%$ from the countryside, $7.0 \%$ from the county/town, and $24.4 \%$ did not report their place of residence. At T2, four weeks later, 185 adolescents ( 96 boys and 89 girls) were re-measured with CAIBQ, with an age range of $12-16(M \pm S D=14.06 \pm 1.06)$. 
Re-measured adolescents differed in age to the first sample $\left[t(1143)=11.58, p<.001, d_{\text {cohen }}=.93\right]$.

To double-check the pattern of missing values on parental marriage status and place of residence, independent $t$ tests were conducted. Results showed that there was no significant difference on social anxiety between participants who reported their parental marriage status and residence place and those who did not report. However, participants who reported their parental marriage and place of residence had significantly lower scores on social negative interpretations than those who did not report $(t(958)$ parental marriage $=2.73, p=.006$, $M_{\text {report }}$ V.s. $M_{\text {not-report }}=2.53$ v.s. $2.71 ; t(958)$ residence place $=$ $2.68, p=.007$. $M_{\text {report }}$ V.s. $M_{\text {not-report }}=2.53$ v.s. 2.71 ). After deleting those who did not report parental marriage status or residence place, similar patterns were found in the reliability and validity analysis. Given this, the total sample $(N=960)$ was used in the following data analyses.

\section{Measures}

Adolescents' Interpretation and Belief Questionnaire (AIBQ), developed by Miers et al. (2008), includes five ambiguous social situations and five non-social situations, and measures adolescents' interpretation bias and their negative belief towards social and non-social situations. The AIBQ presents adolescents with the ten situations in a fixed, random order (see Miers et al., 2008) and asks the respondent to imagine the situation happening to them. Following each situation the respondent rates a positive, negative, and neutral interpretation according to how likely each interpretation would occur in their mind, (1-“Doesn't pop up in my mind", 3-“Might pop up in my mind", 5-“Definitely pops up in my mind"). The three interpretations following each situation were presented in a pre-determined random order to avoid a potential response bias. Finally, respondents were asked to choose which interpretation is the most believable and this provides the measure of belief towards a situation ( $1=$ positive; $2=$ neutral; $3=$ negative). In line with previous studies, the AIBQ variables analyzed in the current study did not include neutral interpretations of social and non-social situations (Giannini \& Loscalzo, 2016; Miers et al., 2008; Yu et al., 2019).

Social Anxiety Scale for Adolescents (SAS-A) was developed and revised by La Greca and Lopez (1998) and, showed that the SAS-A had good psychometric properties. The self-report part of SAS-A is comprised of 18 items and 4 filler items using a 5-point Likert scale (1-"Not at all", 5-“Always"). Zhou et al. (2008) validated the Chinese version of the SAS-A, showing good psychometric properties. The alpha coefficient in the present study was .93.

Short Mood and Feelings Questionnaire (SMFQ), developed by Angold, Costello, Messer, and Winder (1995), is a unifactorial scale and widely used to evaluate depressive symptoms in children and adolescents. Cheng, $\mathrm{Cao}$, and $\mathrm{Su}$
(2009) culturally revised it for Chinese adolescents, and results demonstrated a good reliability and validity. The internal consistency coefficient in the present study was .92 .

\section{Procedure}

The translation and back-translation procedures of the AIBQ were implemented in the following order. First, the first author translated the AIBQ into Chinese, and then discussed with one professor and three $\mathrm{Ph}$.D. candidates from the department of clinical and counselling psychology. After comprehensive discussion, the draft version was confirmed. Second, a professional postgraduate who majored in English and also experienced at translation work but blind to the purpose and original English version of the AIBQ was invited to back-translate. After the back-translation was finished, a few adjustments to the first draft version were made by the first author to be more in line with Chinese individuals' expression habits, and then the back-translator was asked again to modify the wording correspondingly. Then, after several discussions with the original author of the AIBQ, the final Chinese version of AIBQ (C-AIBQ) was confirmed.

One cultural adaption of a negative interpretation did not apply to the Chinese school system. In the original AIBQ, the negative interpretation offered for situation 3 was "This class is too difficult for me; I'll have to repeat a year'. After discussing with the original author of the AIBQ, however, we considered that repeating a year was not so common in Chinese adolescents, therefore, it was substituted with "The subject is too difficult for me; I'll probably fail", which was used by researchers at Oxford University (Booth et al., 2017).

The present study was approved by the Ethics Committee of Beijing Normal University. One middle school from Nanjing city was contacted and participants from four grades (7th, 8th, 10th, and 11th, 983 adolescents in total) agreed to take part in the current study. After the final version of Chinese AIBQ was confirmed, formal measurement process began. To begin with, informed consents were obtained from at least one parent and each participant with the assistance of head teacher of each class. Before commencing, the participants were instructed to ensure a comprehensive understanding of the questionnaires. Then, adolescents were asked to complete the package of questionnaires, including demographic information, C-AIBQ, SAS-A, and SMFQ, in their classroom in the presence of the teacher and one research assistant. After the completion of all measurements, the research assistant thanked the adolescents for their participation. To obtain test-retest reliability of C-AIBQ we approached four classes from 7th and 8th grades whose teacher collaborated with the research team four weeks later, and those classes agreed to re-participate (185 adolescents in total). At the second measurement, only the C-AIBQ was surveyed. 


\section{Data Analyses}

\section{Data Cleaning and Check}

Participants whose missing data exceeded $20 \%$ were excluded from the data analyses $(n=23)$, leaving 960 participants in the final sample with missing data between $.10 \%$ to $3.2 \%$. Although Little's MCAR chi-square test (Little \& Rubin, 2015) was significant $(p<.001)$, further $t$ tests examining the pattern of missing data demonstrated that the variables with missing data were missing at random (MAR), which is acceptable (Rubin, 1976). The missing values of SAS-A, SFMQ, and C-AIBQ (except for negative belief variables), were imputed with Full Information Maximum Likelihood (FIML) estimation via the Expectation Maximization (EM) algorithm (Dempster, Laird, \& Rubin, 2016; Wang \& Deng, 2016) in SPSS 22.0. Regarding the negative belief variable(s) in social and non-social situations, multiple imputation, a default method in SPSS via logistic regression, was used to deal with its missing data.

One-sample K-S nonparametric tests showed that the data (including SAS-A, SFMQ, and C-AIBQ subscales) was not normally distributed $(p s<.001$, Skewness $=-.67 \sim 1.28$, Kurtosis $=-.40 \sim 3.64$ ). However, Curran, West, and Finch (1996) reported that the absolute value of skewness and kurtosis less than 2.0 and 7.0, respectively, would be acceptable, with no data transformation necessary.

\section{Validity and Reliability Examination}

First, to test the construct validity of the C-AIBQ confirmatory factor analysis (CFA) was performed using Mplus 7.11. CFA is used to study the relationships between a set of observed variables and a set of continuous latent variables (Muthén \& Muthén, 2012). In the present study, to examine the degree to which the C-AIBQ items measure the intended negative and positive interpretations of social and non-social situations, a CFA model including negative and positive interpretation in social (abbr., SocNeg, SocPos) and non-social (abbr., NonsocNeg, NonsocPos) situations, respectively, was tested, with four latent factors. For each latent factor (i.e., SocNeg, SocPos, NonsocNeg, or NonsocPos), 5 items were included. In this model, the four factors were allowed to correlate with each other. Next, in line with previous psychometric studies (e.g., Gutiérrez et al., 2017; Sliwinski et al., 2018), we examined bivariate correlations among the C-AIBQ subscales. As the score on negative belief in social and non-social situations, technically, was categorical, ${ }^{1}$ Pearson correlation for

\footnotetext{
${ }^{1}$ Although the fact that answer response to belief question is, technically, categorical, the items still could be summed and averaged for social and non-social belief subscales (Miers et al., 2008; Yu et al., 2019).
}

continuous variables was inapplicable. Thus, we computed Spearman rank correlations between negative belief subscales and the positive interpretation and negative interpretation in social and non-social situations. To compare the size of the relations between the C-AIBQ variables, and later the correlations among AIBQ-C subscales and social anxiety and depression, William's tests were conducted (Diedenhofen \& Musch, 2015).

Second, internal consistency reliability of the positive and negative social and non-social subscales was assessed with Cronbach's alpha. As the score on negative belief in social and non-social situations was categorical, Cronbach's alpha for continuous variables was inapplicable. Therefore, for the categorical belief variables, following McDonald (1999), omega coefficients $^{2}$ via the variance approach was adopted to compute internal consistency reliability. Test-retest reliability was assessed using intra-class correlation (ICC) ${ }^{3}$ for the positive and negative social and non-social subscales. For the categorical belief subscales Kappa coefficients were adopted (Karras, 1997; Kraemer, Periyakoil, \& Noda, 2002). As a measure of reliability, the criteria of kappa coefficient values were as follows: $<.20$ as slight, $.20-.40$ as fair, $.40-.60$ as moderate, $.60-.80$ as substantial, $>.80$ as perfect (Landis \& Koch, 1977).

Third, social anxiety level as assessed with the SAS-A was used for measuring convergent validity, and depression level as assessed with the SMFQ for discriminant validity. Partial correlations between social anxiety and the C-AIBQ subscales were computed whilst controlling for the influence of depression, and vice versa. All analyses, except for kappa coefficients, ${ }^{4}$ were conducted in SPSS 22.0 and Mplus 7.11 software.

\section{Results}

\section{Descriptive Statistics of the C-AIBQ, SAS-A, and SMFQ Variables}

The mean and standard deviation for each variable in the general sample and grouped by gender are shown in Table 1. As can be seen, in the total sample non-social situations had higher negative and positive ratings compared to social situations. Girls generally showed higher negative interpretations towards social and non-social situations, positive interpretations towards non-social situations, social anxiety and depression level as compared to boys.

\footnotetext{
${ }^{2}$ Omega coefficient $(\omega)$ is one of the indicators to assess the consistency (Revelle \& Zinbarg, 2009).

${ }^{3}$ Single measure ICC was adopted in the present study, which means the stability of scores from one rater measured at two different occasions.

${ }^{4}$ Kappa coefficients analyses were performed using MedCalc for Windows, version 19.0.3 (MedCalc Software, Ostend, Belgium).
} 
Table 1 Descriptive statistics of all study variables for the whole sample and by gender

\begin{tabular}{llll}
\hline & \multicolumn{3}{l}{ Mean $(S D)$} \\
\cline { 2 - 3 } & Total Sample & Boys & Girls \\
\hline SocNeg & $2.58(.90)$ & $2.46(.89)$ & $2.70(.89)$ \\
NonsocNeg & $2.61(.74)$ & $2.52(.79)$ & $2.71(.67)$ \\
SocPos & $2.26(.75)$ & $2.36(.79)$ & $2.14(.68)$ \\
NonsocPos & $3.27(.87)$ & $3.14(.94)$ & $3.42(.76)$ \\
social Anxiety & $49.74(16.19)$ & $46.97(16.50)$ & $52.93(15.27)$ \\
Depression & $9.32(6.42)$ & $8.46(6.33)$ & $10.25(6.40)$ \\
\hline
\end{tabular}

Note. SocNeg = Negative interpretation in social situations, NonsocNeg $=$ Negative interpretation in non-social situations, SocPos $=$ Positive interpretation in social situations, NonsocPos $=$ Positive interpretation in non-social situations. ${ }^{* * * *} p<.001$

\section{Construct Validity of C-AIBQ Variables}

CFA results demonstrated good model fit indexes for negative interpretations and positive interpretations both in social situations and non-social situations, $\chi^{2}(1)=53.72, p<.001$, $\mathrm{CFI}=.99, \mathrm{TLI}=.93, \mathrm{RMSEA}=.096, \mathrm{SRMR}=.02 .{ }^{5}$ The standardized factor loadings of the four subscales, SocNeg, NonsocNeg, SocPos and NonsocPos, were .79, .77, 48, and .74 , respectively.

As shown in Table 2, the inter-correlations of (negative and positive) interpretations in social and non-social situations were significant and positive $(r \mathrm{~s}=.12$ to $.58, p \mathrm{~s}<.001)$, respectively, at a low to medium level. In addition, Spearman correlation results showed that, social negative belief showed positive association with social negative interpretation $\left(r_{s-}\right.$ $=.45, p<.001$ ), and non-social negative belief also demonstrated positive correlation with non-social negative interpretation $\left(r_{s}=.19, p<.001\right)$. Social negative belief negatively correlated with positive interpretation $\left(r_{s}=-.33, p<.001\right)$, and non-social belief negatively associated with positive interpretations in non-social situations $\left(r_{s}=-.44, p<.001\right)$.

To examine the consistency of interpretations across situations, several particular comparisons were made. First, the relationship between SocNeg and NonsocNeg was significantly stronger than that between SocNeg and SocPos, $t(957)=13.78, p<.001$, and the correlation between NonsocNeg and SocNeg was significantly stronger than the association between NonsocNeg and NonsocPos, $t(957)=$ $6.83, p<.001$. Second, the relationship between NonsocPos and NonsocNeg was not stronger than that between NonsocPos and SocPos $[t(957)=-.28, p=.609]$.

\footnotetext{
${ }^{5}$ Notwithstanding the value of RMSEA is greater than .08, the cut-off which is suggested by SEM investigators, the values of CFI, TLI and SRMR are good; hence, overall, the model is still acceptable (Barrett, 2007).
}

Table 2 Inter-correlations of C-AIBQ variables $(N=960)$

\begin{tabular}{llllll}
\hline & & 1 & 2 & 3 & 4 \\
\hline 1 & SocNeg & - & & & \\
2 & NonsocNeg & $.58^{* * *}$ & - & \\
3 & SocPos & $.12^{* * *}$ & $.23^{* * *}$ & - \\
4 & NonsocPos & $.32^{* * *}$ & $.37^{* * *}$ & $.38^{* * *}$ & - \\
\hline
\end{tabular}

Note. SocNeg = Negative interpretation in social situations, NonsocNeg $=$ Negative interpretation in non-social situations, SocPos $=$ Positive interpretation in social situations, NonsocPos $=$ Positive interpretation in non-social situations. ${ }^{* * * *} p<.001$

\section{Internal Consistency and Test-Retest Coefficients of C- AIBQ Variables}

As shown in Table 3, the Cronbach's $\alpha$ coefficients of SocNeg, NonsocNeg, SocPos and NonsocPos were .54 to .74 , respectively. The ICCs of SocNeg, NonsocNeg, SocPos and NonsocPos were .39 to .51 . Results from variance approach for examining the internal consistency reliability showed that, for belief subscale in social situations, $\omega=.88$; with regard to non-social belief subscale, $\omega=.87$. The kappa coefficient for social and non-social situations, respectively, was .21 (95\% CI $[-.23, .65])$, and $.53(95 \%$ CI $[.37, .69])$. Albeit the kappa coefficient of social belief subscale was not at significant level, it was still greater than the suggested fair value, .20 .

\section{Convergent and Discriminant Validity of C-AIBQ Variables}

Table 4 displays the convergent and discriminant correlations between C-AIBQ variables and social anxiety (controlling for depression), and C-AIBQ variables and depression (controlling for social anxiety). In general, social negative interpretations had a significant correlation with social anxiety $\left(r_{p}=.36\right.$, $p<.001)$ and depression $\left(r_{p}=.24, p<.001\right)$. Importantly, social negative interpretations showed a stronger correlation with social anxiety than that with depression $(t(957)=3.93$,

Table 3 Internal consistency and test-retest coefficients of C-AIBQ variables

\begin{tabular}{lllll}
\hline & SocNeg & NonsocNeg & SocPos & NonsocPos \\
\hline Cronbach's $\alpha$ coefficient & .74 & .54 & .59 & .63 \\
ICC $^{\text {a }}$ & .51 & .45 & .48 & .39 \\
\hline
\end{tabular}

Note. SocNeg = Negative interpretation in social situations, NonsocNeg = Negative interpretation in non-social situations, SocPos = Positive interpretation in social situations, NonsocPos = Positive interpretation in non-social situations. ${ }^{a}$. ICC is based on a different sized sample $(N=185)$ to compute test-retest reliability 
Table 4 The relations between C-AIBQ variables with social anxiety and depression, respectively $(N=960)$

\begin{tabular}{llll}
\hline C-AIBQ variables & social Anxiety $^{\mathrm{a}}$ & Depression $^{\mathrm{b}}$ & $t$ (William's test) \\
\hline SocNeg & $.36^{* * * *}$ & $.24^{* * *}$ & $3.93^{* * *}$ \\
NonsocNeg & $.28^{* * * *}$ & $.10^{* * *}$ & $5.74^{* * * *}$ \\
SocPos & .01 & -.02 & .92 \\
NonsocPos & $.24^{* * *}$ & $-.07^{*}$ & $10.03^{* * *}$ \\
\hline
\end{tabular}

Note. SAS-A = Social Anxiety Scale for Adolescents, SMFQ = Short Mood and Feelings Questionnaire, SocNeg $=$ Negative interpretation in social situations, NonsocNeg = Negative interpretation in non-social situations, SocPos $=$ Positive interpretation in social situations, NonsocPos $=$ Positive interpretation in non-social situations. ${ }^{a}$. Controlling for social anxiety; ${ }^{\mathrm{b}}$. Controlling for depression.

${ }^{*} p<.05,{ }^{* *} p<.01,{ }^{* * *} p<.001$.

$p<.001)$. Moreover, the association between social negative interpretation and social anxiety was significantly stronger than that between non-social negative interpretation and social anxiety $(t(957)=2.90, p=.002)$. Social positive interpretations neither had a significant correlation with social anxiety, nor with depression. Regarding non-social situations, negative interpretations were significantly associated both with social anxiety $\left(r_{p}=.28, p<.001\right)$ and depression $\left(r_{p}=.10, p=.003\right)$, but the correlation was higher with social anxiety than depression $(t(957)=5.74, p<.001)$. Non-social positive interpretations significantly and positively correlated with social anxiety $\left(r_{p}=.24, p<.001\right)$, but correlated negatively with depres$\operatorname{sion}\left(r_{p}=-.07, p=.028\right)$; a significant difference between correlations $(t(957)=10.03, p<.001)$.

\section{Discussion}

The current study's aim was to examine the psychometric properties of the C-AIBQ in Chinese adolescents. The findings supported a four-factor model of negative and positive interpretation in social and non-social situations. In general, the C-AIBQ subscales showed modest to acceptable reliability and validity. Particularly, the negative interpretation subscale for social situations showed good construct validity, acceptable internal consistency reliability, convergent and discriminant validity and test-retest reliability.

First, results from the CFA demonstrated a good construct validity of negative interpretations and positive interpretations in C-AIBQ for both social and non-social situations in Chinese adolescents. Therefore, structurally, this would suggest that there existed a good relationship among negative and positive interpretations, and also showed a consistency between interpretations and situations. Regarding the correlation analyses among C-AIBQ variables, the inter-correlations of (negative and positive) interpretations in social and nonsocial situations were shown to be at a moderate level, except for the association between SocNeg and SocPos. That negative belief showed positive correlation with negative interpretation and negative association with positive interpretation, respectively, indicated that the higher level of negative belief Chinese adolescents had, the more likely it was for them to negatively interpret the scenarios, which is line to the cognitive model for social anxiety disorder (e.g., Clark \& Wells, 1995). Negative interpretation significantly and positively correlated with positive interpretation, both in the context of social and non-social situations. A possible explanation could be that negative and positive interpretation are not opposite, but, independent constructs (Huppert, Foa, Furr, Filip, \& Mathews, 2003; Huppert, Pasupuleti, Foa, \& Mathews, 2007). Therefore, it's probable that both negative and positive interpretations exist simultaneously. The William's tests showed that individuals with the tendency to negatively interpret social (or non-social) situations would be more likely to threateningly interpret non-social (or social) situations. This would suggest a stability and consistency of negative interpretations across situation type.

Second, with regard to internal reliability, positive interpretations and negative interpretations in social and non-social situations, respectively, showed moderate to good correlations. In particular, the negative interpretation subscale for social situations showed good internal consistency reliability which was similar to previous studies $(\alpha=.65$, Loscalzo \& Giannini, 2015; $\alpha=.73$, Miers et al., 2013). ICC results demonstrated that, negative and positive interpretation for social and non-social situations assessed at the first time significantly correlated with that assessed at the second time, which suggests moderate test-retest reliability. Variance approach results and kappa coefficients also suggested that negative belief in social and non-social situations showed a fair to moderate reliability.

Third, of the C-AIBQ subscales, social negative interpretation, followed by non-social negative interpretation, showed the largest correlation with social anxiety and depression, respectively. That NonsocNeg showed the second largest association with social anxiety might indicate that non-social situations actually include a "social element". For instance, in situation 8 "Bike", socially anxious persons might not only think "It has been stolen", but also "Everyone is looking at me; it so stupid of me that I can't find my bike". Notwithstanding that it is difficult to completely take the "social element" out of non-social situations, after conducting William's test, the relationship between social negative interpretation and social anxiety was still significantly stronger than that between non-social negative interpretation and depression. Moreover, the relationship between social negative interpretation and social anxiety was significantly stronger than that between social negative interpretation and 
depression. Likewise, the correlation between non-social negative interpretation and social anxiety was stronger than that between non-social negative interpretation and depression. Non-social positive interpretation showed a negative correlation with depression. This finding is in line with the Cognitive Model of Depression, which states that depressed individuals block positive stimuli or memories because of a systematic cognitive bias (Beck, 2008). However, positive interpretation of non-social situations displayed a positive correlation with social anxiety. A possible reason could be that, as previously explained, being a separate construct from negative interpretation (Huppert et al., 2003, 2007), positive interpretation might occur alongside social anxiety symptoms in nonsocial situations.

The cognitive model for SAD has pointed out the important role of interpretation bias in the occurrence and maintenance of social anxiety (Clark \& Wells, 1995), and can be extended to adolescents (Leigh \& Clark, 2018). Given this, the present study comprehensively examined the psychometric properties of the C-AIBQ, a measure of interpretation bias in a large Chinese adolescent sample. The availability of this measure makes the provision of cultural evidence and comparison possible. In addition, a recent review has indicated that, for socially anxious adolescents, interpretation bias modification training has been found to be a potential tool to improve interpretation bias and negative emotion (Biagianti, Conelea, Brambilla, \& Bernstein, 2020). The remission of interpretation bias could further enhance individuals' self-image and self-esteem (Pictet, 2014), and improve their anxiety sensitivity (MacDonald, Koerner, \& Antony, 2013). Therefore, the CAIBQ could be beneficial as an instrument to measure whether the above-mentioned intervention could alleviate Chinese adolescents' social fear by targeting interpretation bias.

Albeit above-mentioned advantages, we acknowledge that there are a few weaknesses in the psychometric properties of the C-AIBQ, particularly regarding the not-very-satisfactory testretest reliability of the social belief subscale and relatively low internal consistency of negative interpretations in non-social situations and positive interpretations in social situations. Then, as for the test-retest reliability, convenience sampling was used. However, the findings overall demonstrated moderate reliability and validity, especially SocNeg, appearing a helpful tool for assessing interpretations of ambiguous situations in Chinese adolescents. We acknowledge that, based on our sample, it is not possible to conclude that the AIBQ-C is ready for clinical research in China. Nevertheless, considering previous findings from Italian adolescents diagnosed with SAD and assessed with the AIBQ in which a clinical cut-off interpretation bias score was suggested (Loscalzo et al., 2017), we recommend that future research could focus on the social negative interpretation subscale as a potential identifying characteristic of clinical social anxiety and identify cut-off scores with SAD diagnosed Chinese adolescents.
Acknowledgements We are so appreciative of Dr. Chenying Zhao for providing information and suggestion for data processing.

Code Availability Not applicable.

Authors' Contribution Meng Yu designed the research, deal with data, and wrote the manuscript. P. Michiel Westenberg structured and critically revised the manuscript. Ye Wang helped collect data. Jianping Wang designed and consulted the research. Anne C. Miers structured and critically revised the manuscript, and provided ideas to data processing.

Funding This work was supported by the China Scholarship Council under Grant [2017]3109.

Data Availability When contacting first or corresponding author, they will be accessible to the data and material.

\section{Compliance with Ethical Standards}

Conflict of Interest No potential conflict of interest was reported by all authors.

Ethics Approval This study was approved by affiliations' ethical institution board of corresponding authors' university and all procedures was in accordance with the ethical standards of 1964 Helsinki declaration.

Informed Consent Informed consent was obtained from all participants and at least their single parent.

\section{References}

American Psychiatric Association (Ed.). (2013). Diagnostic and statistical manual of mental disorders, 5th ed. Washington, DC: American Psychiatric Association.

Angold, A., Costello, E. J., Messer, S. C., \& Winder, F. (1995). Development of a questionnaire for use in epidemiological studies of depression in children and adolescents. International Journal of Methods in Psychiatric Research, 5(4), 237-249. https://doi.org/ 1049-8931.95/040237-13.

Barrett, P. (2007). Structural equation modelling: Adjudging model fit. Personality and Individual Difference, 42(5), 815-824. https://doi. org/10.1016/j.paid.2006.09.018.

Beck, A. (2008). The evolution of the cognitive model of depression and its neurobiological correlates. American Journal of Psychiatry, 165(8), 969-977. https://doi.org/10.1176/appi.ajp.2008.08050721.

Biagianti, B., Conelea, C., Brambilla, P., \& Bernstein, G. (2020). A systematic review of treatments targeting cognitive biases in socially anxious adolescents. Journal of Affective Disorders, 264, 543-551. https://doi.org/10.1016/j.jad.2019.12.002.

Blöte, A. W., \& Westenberg, P. M. (2007). Socially anxious adolescents' perception of treatment by classmates. Behaviour Research and Therapy, 45(2), 189-198. https://doi.org/10.1016/j.brat.2006.02. 002.

Blöte, A, W., Miers, A, C., Heyne, D, A., \& Westenberg, P, M. (2015). Social anxiety and the school environment of adolescents. In K. Ranta, A. M. La Greca, L.-J. Garcia-Lopez, \& M. Marttunen (Eds.), Social Anxiety and Phobia in Adolescents. Switzerland: Springer International Publishing. https://doi.org/10.1007/978-3319-16703-9 1.

Booth, C., Songco, A., Parsons, S., Heathcote, L., Vincent, J., Keers, R., \& Fox, E. (2017). The CogBIAS longitudinal study protocol: Cognitive and genetic factors influencing psychological functioning 
in adolescence. BioMed Central Psychology, 5(1), 1-14. https://doi. org/10.1186/s40359-017-0210-3.

Cheng, P., Cao, F., \& Su, L. (2009). Reliability and validity of the short mood and feelings questionnaire in Chinese adolescent. Chinese Mental Health Journal, 23(1), 60-63. https://doi.org/10.3969/j. issn.1000-6729.2009.01.016.

Clark, D. M., \& Wells, A. (1995). A cognitive model of social phobia. In R. G. Heimberg, M. R. Liebowitz, D. A. Hope, \& F. R. Schneier (Eds.), Social phobia: Diagnosis, assessment, and treatment. New York, NY: The Guildford Press.

Creswell, C., Murray, L., \& Cooper, P. (2014). Interpretation and expectation in childhood anxiety disorders: Age effects and social specificity. Journal of Abnormal Child Psychology, 42(3), 453-465. https://doi.org/10.1007/s10802-013-9795-z.

Curran, P. J., West, S. G., \& Finch, J. F. (1996). The robustness of test statistics to nonnormality and specification error in confirmatory factor analysis. Psychological Methods, 1(1), 16-29. https://doi. org $/ 10.1037 / 82-989 X / 96 / \$ 3.00$

Dempster, A. P., Laird, N. M., \& Rubin, D. B. (2016). Maximum likelihood from incomplete data via the EM algorithm. Journal of the Royal Statistical Society, 39(1), 1-38.

Diedenhofen, B., \& Musch, J. (2015). Cocor: A comprehensive solution for the statistical comparison of correlations. PLoS One, 10(4), 112. https://doi.org/10.1371/journal.pone.0121945.

Fu, X., Du, Y., Au, S., \& Lau, J. Y. F. (2013). Reducing negative interpretations in adolescents with anxiety disorders: A preliminary study investigating the effects of a single session of cognitive bias modification training. Developmental Cognitive Neuroscience, 4, 29-37. https://doi.org/10.1016/j.den.2012.11.003.

Giannini, M., \& Loscalzo, Y. (2016). Social anxiety and adolescence: Interpretation bias in an Italian sample. Scandinavian Journal of Psychology, 57(1), 65-72. https://doi.org/10.1111/sjop.12263.

Gutiérrez, F., Aluja, A., Peri, J. M., Calvo, N., Ferrer, M., Baillés, E., et al. (2017). Psychometric properties of the Spanish PID-5 in a clinical and a community sample. Assessment, 24(3), 326-336. https://doi. org/10.1177/1073191115606518.

Hirsch, C. R., Meeten, F., Krahé, C., \& Reeder, C. (2016). Resolving ambiguity in emotional disorders: The nature and role of interpretation biases. Annual Review of Clinical Psychology, 12(1), 281-305. https://doi.org/10.1146/annurev-clinpsy-021815-093436.

Hodson, K. J., McManus, F. V., Clark, D. M., \& Doll, H. (2008). Can Clark and Wells' (1995) cognitive model of social phobia be applied to young people? Behavioural and Cognitive Psychotherapy, 36(4), 449-461. https://doi.org/10.1017/S1352465808004487.

Huppert, J. D., Foa, E. B., Furr, J. M., Filip, J. C., \& Mathews, A. (2003). Interpretation bias in social anxiety: A dimensional perspective. Cognitive Therapy and Research, 27(5), 569-577. https://doi.org/ 10.1023/A:1026359105456.

Huppert, J. D., Pasupuleti, R. V., Foa, E. B., \& Mathews, A. (2007). Interpretation biases in social anxiety: Response generation, response selection, and self-appraisals. Behaviour Research and Therapy, 45(7), 1505-1515. https://doi.org/10.1016/j.brat.2007.01. 006.

Karras, D. J. (1997). Statistical methodology: II. Reliability and validity assessment in study design, Part A. Academic Emergency Medicine, 4(1), 64-71.

Kessler, R. C., Mcgonagle, K. A., Zhao, S., Nelson, C. B., Hughes, M., Eshleman, S., et al. (1994). Lifetime and 12-month prevalence of DSM-III-R psychiatric disorders in the United States. Archives of General Psychiatry, 51(1), 8-19. https://doi.org/10.1001/archpsyc. 1994.03950010008002.

Kraemer, H. C., Periyakoil, V. S., \& Noda, A. (2002). Kappa coefficients in medical research. Statistics in Medicine, 2129(April), 2109-2129. https://doi.org/10.1002/sim.1180.

La Greca, A. M., \& Lopez, N. (1998). Social anxiety among adolescents : Linkages with peer relations and friendships. Journal of Abnormal
Child Psychology, 26(2), 83-94. https://doi.org/0091-0627/98/ 0400-0083\$15.00/0.

Landis, J. R., \& Koch, G. G. (1977). The measurement of observer agreement for categorical data. Bioetrics, 33, 159-174.

Leigh, E., \& Clark, D. M. (2018). Understanding social anxiety disorder in adolescents and improving treatment outcomes: Applying the cognitive model of Clark and Wells (1995). Clinical Child and Family Psychology Review, 21, 388-414. https://doi.org/10.1007/ s10567-018-0258-5.

Little, R. J., \& Rubin, D. B. (2015). Missing Data. International Encyclopedia of the Social \& Behavioral Sciences, 15, 602-607. https://doi.org/10.1016/B978-0-08-097086-8.42082-9.

Loscalzo, Y., \& Giannini, M. (2015). Prevention of social anxiety disorder in adolescence. Psychometric properties of adolescents' interpretation and belief questionnaire (AIBQ). Italian Journal of Research and Intervention, 8(2), 1-6.

Loscalzo, Y., Giannini, M., \& Miers, A. C. (2017). Social anxiety and interpretation bias: Examining clinical and subclinical components in adolescents. Child and Adolescent Mental Health., 23, 169-176. https://doi.org/10.1111/camh.12221.

MacDonald, E. M., Koerner, N., \& Antony, M. M. (2013). Modification of interpretive bias: Impact on anxiety sensitivity, information processing and response to induced bodily sensations. Cognitive Therapy and Research, 37(4), 860-871. https://doi.org/10.1007/ s10608-012-9519-7.

McDonald, R. P. (1999). A unified treatment. Mahwah, New Jersey: Lawrence Erlbaum Assoicates.

Meacham, J. A., \& Santilli, N. R. (1982). Interstage relationships in Erikson' s theory: Identity and intimacy. Child Development, 53(6), 1461-1467.

Miers, A. C., Blöte, A. W., Bögels, S. M., \& Westenberg, P. M. (2008). Interpretation bias and social anxiety in adolescents. Journal of Anxiety Disorders, 22(8), 1462-1471. https://doi.org/10.1016/j. janxdis.2008.02.010

Miers, A. C., Blöte, A. W., Rooij, M. D., Bokhorst, C. L., \& Westenberg, P. M. (2013). Trajectories of social anxiety during adolescence and relations with cognition, social competence, and temperament. Journal of Abnormal Child Psychology, 41, 97-110. https://doi. org/10.1007/s10802-012-9651-6.

Miers, A. C., Sumter, S. R., Clark, D. M., \& Leigh, E. (2020). Interpretation bias in online and offiline social environments and associations with social anxiety, peer victimization, and avoidance behavior. Cognitive Therapy and Research, 44, 820-833. https:// doi.org/10.1007/s10608-020-10097-1.

Mueller, S, C., Unal, C., Saretta, M., Mughairbi, F, A., Gómez-Odriozola, J., Calvete, E., \& Metin, B. (2020). Working memory and emotional interpretation bias in a sample of Syrian refugee adolescents. European Child \& Adolescent Psychiatry, https://doi.org/10.1007/ s00787-020-01656-8.

Muthén, L. K., \& Muthén, B. O. (2012). Mplus User's Guide (Seventh Edition). Los Angeles, CA: Muthén \& Muthén.

Pictet, A. (2014). Looking on the bright side in social anxiety: The potential benefit of promoting positive mental imagery. Frontiers in Human Neuroscience, 8, 1-5. https://doi.org/10.3389/fnhum.2014. 00043.

Reuland, M. M., \& Teachman, B. A. (2014). Interpretation bias modification for youth and their parents: A novel treatment for early adolescent social anxiety. Journal of Anxiety Disorders, 28(8), 851864. https://doi.org/10.1016/j.janxdis.2014.09.011.

Revelle, W., \& Zinbarg, R. E. (2009). Coefficients alpha, beta, omega and the glb: Comments on Sijtsma. Psychometrika, 74(1), 145-154. https://doi.org/10.1007/s11336-008-9102-z.

Rubin, D. B. (1976). Inference and missing data. Biometrika, 61, 581592.

Sfärlea, A., Buhl, C., Loechner, J., Neumüller, J., Thomsen, L. A., Starman, K., et al. (2020). "I am a Total...loser"-the role of 
interpretation bias in youth depression. Journal of Abnormal Child Psychology, 48, 1337-1350. https://doi.org/10.1007/s10802-02000670-3.

Sliwinski, M., Mogle, J. A., Hyun, J., Munoz, E., Smyth, J. M., \& Lipton, R. B. (2018). Reliability and validity of ambulatory cognitive assessments. Assessment, 25(1), 14-30. https://doi.org/10.1177/ 1073191116643164.

Songco, A., Booth, C., Spiegler, O., Parsons, S., \& Fox, E. (2020). Anxiety and depressive symptoms trajectories in adolescence and the co-occurring development of cognitive biases: Evidence from the CogBIAS longitudinal study. Journal of Abnormal Child Psychology, 48, 1617-1633. https://doi.org/10.1007/s10802-02000694-9.

Wang, M. C., \& Deng, Q. W. (2016). The mechanism of auxiliary variables in full information maximum likelihood-based structural equation models with missing data. Acta Psychologica Sinica, 48(11), 1489-1498. https://doi.org/10.3724/SP.J.1041.2016.01489.
Yang, J. (2011). Interpretation bias in adolescents with social anxiety (Master's thesis). Southwest University.

Yu, M., Westenberg, P. M., Li, W., Wang, J., \& Miers, A. C. (2019). Cultural evidence for interpretation bias as a feature of social anxiety in Chinese adolescents. Anxiety, Stress, \& Coping, 32(4), 376-386. https://doi.org/10.1080/10615806.2019.1598556.

Zhou, X., Xu, Q., Inglés, C. J., Hidalgo, M. D., \& La Greca, A. M. (2008). Reliability and validity of the Chinese version of the social anxiety scale for adolescents. Child Pyschiatry and Human Development, 39(2), 185-200. https://doi.org/10.1007/s10578007-0079-0.

Zhu, S. (2016). The Relatinships among parental style and interpretation bias on social anxiety in child aged 11-15 (Master's thesis). Zhejiang Sci-Tech University.

Publisher's Note Springer Nature remains neutral with regard to jurisdictional claims in published maps and institutional affiliations. 